\title{
TWO APPLICATIONS OF TWISTED WREATH PRODUCTS TO FINITE SOLUBLE GROUPS
}

\author{
BY \\ TREVOR O. HAWKES
}

\begin{abstract}
The group construction sometimes known as the twisted wreath product is used here to answer two questions in the theory of finite, soluble groups: first to show that an arbitrary finite, soluble group may be embedded as a subgroup of a group whose upper nilpotent series is a chief series; second to construct an $A$-group whose Carter subgroup is "small" relative to its nilpotent length.
\end{abstract}

1. Some preliminaries. We begin with a few elementary remarks about nilpotent length. The largest nilpotent normal subgroup of a group $G$, usually known as the Fitting subgroup, is denoted by $F(G)$. The upper nilpotent series $\left\{F_{i}(G)\right\}$ is defined recursively thus: $F_{1}(G)=F(G)$ and $F_{i+1}(G) / F_{i}(G)=$ $F\left(G / F_{i}(G)\right)$ for $i \geqslant 1$. The smallest integer $l(=l(G))$ such that $F_{l}(G)=G$ is called the nilpotent length of $G$. If $L(G)$ denotes the nilpotent residual of $G$, i.e. the smallest normal subgroup of $G$ with nilpotent quotient, the lower nilpotent series $\left\{L_{i}(G)\right\}$ of $G$ is defined recursively by setting $L_{1}(G)=L(G)$ and $L_{i+1}(G)=L\left(L_{i}(G)\right)$ for $i \geqslant 1$. The invariant $l(G)$ is the smallest $l$ such that $L_{l}(G)=1$ and may also be characterised as the smallest number of factors that can appear in any normal series with nilpotent factors.

Next we give the details of the twisted wreath product construction and its associated notation (cf. [6, I, 15.10]). Let $X$ and $G$ be groups. Let $H$ be a subgroup of $G$ of finite index, $T$ a right transversal to $H$ in $G$, and $\alpha$ a homomorphism from $H$ into $\operatorname{Aut}(X)$. Denote by $X^{T}$ the set of maps $f: T \rightarrow X$. Then the twisted wreath product of $X$ with $G$ for $(H, \alpha)$ has as its underlying set

$$
W=\left\{(f, g): f \in X^{T}, g \in G\right\} .
$$

If $h \in H$ and $x \in X$, denote the image of $x$ under the automorphism $\alpha(h)$ by $x^{h}$. If $t \in T$ and $g_{1} \in G$, we have

$$
\operatorname{tg}_{1}=h t^{\prime}
$$

Received by the editors November 4, 1974.

AMS (MOS) subject classifications (1970). Primary 20D10, 20F25; Secondary $20 \mathrm{C1}$.

Key words and phrases. Twisted wreath product, nilpotent length, Clifford theory, primitive group, Carter subgroup. 
for uniquely determined $h \in H$ and $t^{\prime} \in T$. The binary operation making $W$ into a group is then given by

$$
\left(f_{1}, g_{1}\right)\left(f_{2}, g_{2}\right)=\left(f, g_{1} g_{2}\right)
$$

where $f(t)=f_{1}(t) f_{2}\left(t^{\prime}\right)^{h^{-1}}$ and $h$ is given by equation (1. $\left.\alpha\right)$. Direct calculation shows that this binary operation is associative, and the identity of $W$ is $(e, 1)$, where $e \in X^{T}$ is defined by: $e(t)=1$ for all $t \in T$. If $f^{*} \in X^{T}$ is defined by $f^{*}\left(t^{\prime}\right)=\left(f(t)^{-1}\right)^{h}$ whenever $t g=h t^{\prime}$, the element $\left(f^{*}, g^{-1}\right)$ is the inverse of $(f, g)$. Accordingly $W$ is a group, and we shall denote it by the symbol $X \sim_{(H, \alpha)} G$. We shall make the obvious identification of $G$ with the subgroup $\{(e, g): g \in G\}$ of $W$. The subgroup $B=\left\{(f, 1): f \in X^{T}\right\}$ is called the base group of $W$. It is a normal subgroup of $W$ isomorphic with a direct product of $|T|$ copies of $X$, and $W$ is a semidirect product of $B$ with $G$. If $T=\left\{t_{1}, t_{2}, \ldots, t_{n}\right\}$ we shall sometimes use the notation $\left(x_{1}, x_{2}, \ldots, x_{n}\right)$ for the element $(f, 1)$ of $B$ for which $f\left(t_{i}\right)=x_{i}$. Furthermore, if $Y$ is a subgroup of $X$ and $T_{0}$ a subset of $T$, we shall use the notation $Y\left(T_{0}\right)$ for the subgroup $\{(f, 1): f(t) \in Y$ for $t \in T_{0}, f(t)=1$ for $\left.t \in T \backslash T_{0}\right\}$ of $B$; thus, for example, $B$ is $X(T)$ in this notation. We end this discussion of the twisted wreath product with two elementary observations about special cases:

(1.1) Let $H$ be a subgroup of $G$ of finite index, and let $X$ be a $k H$-module for some field $k$. Associated with the H-action on $X$ there is a homomorphism $\alpha$ from $H$ into the group of nonsingular, $k$-linear transformations of $X$. If $X$ is regarded as an abelian group and written multiplicatively, $\alpha$ maps $H$ into $\operatorname{Aut}(X)$, and we can form the twisted wreath product $X \sim_{(H ; \alpha)} G$ as above. Then the base group of the wreath product, now written additively and viewed again as $a k$-space on which $G$ acts, is isomorphic with the induced $k G$-module $X^{G}$.

REMARK. It should be clear from the context whether the notation $A^{B}$ means a Cartesian product or an induced module.

(1.2) If there exists a subgroup $L$ complementary to $H$ in $G$ (i.e. a subgroup satisfying $H L=G$ and $H \cap L=1$ ), and if we take $T=L$ for the transversal to $H$ in the twisted wreath product $X \sim_{(H, \alpha)} G$, then $B L \cong X \sim_{\text {reg }} L$, the standard wreath product with respect to the regular permutation representation of $L$.

The next result on induced representations is an application of Clifford's theorem (see [6, V, Hauptsatz 17.3]).

(1.3) Let $N$ be an abelian normal subgroup of a finite group $G$ such that $N \nless Z(G)$. Assume that $G$ contains a nonnormal, maximal subgroup $M$ which contains $N$ and a normal subgroup $K$ with the following properties:

(i) $M / K$ is cyclic;

(ii) $N K=M$; 
(iii) $\operatorname{core}_{G}(K)=1$.

Let $k$ be a field of characteristic not dividing $|M: K|$ and let $U$ be an irreducible $k M$-module with $\operatorname{ker}(M$ on $U)=K$. Then the induced module $U^{G}$ is irreducible and faithful for $G$.

REMARKS. 1. The existence of such a module $U$ is ensured by assumption (i) and the assumption that $\operatorname{char}(k)$ does not divide $|M: K|$. 2. The subgroup core $_{G}(K)$ is by definition the intersection of the $G$-conjugates of $K$, or, equivalently, the largest normal subgroup of $G$ contained in $K$.

Proof. Let $V$ denote the $k G$-module $U^{G}$. Let $L=\operatorname{ker}(G$ on $V) \preccurlyeq G$. If $L \nless M$, we have $L M=G$ since $M$ is maximal, and therefore $L \cap M<L$; then by Mackey's theorem $V_{L} \simeq\left(U_{L \cap M}\right)^{L}$, a nontrivial $L$-module. This contradiction implies that $L \leqslant M$. In particular, we have $L \leqslant \operatorname{ker}(M$ on $V) \leqslant$ $\operatorname{ker}(M$ on $U)=K$. Therefore $L \leqslant \operatorname{core}_{G}(K)=1$ by assumption (iii), and $V$ is faithful for $G$.

It remains to prove that $V$ is irreducible. First observe that by assumption (ii) $U_{N}$ is irreducible and therefore appears as a direct summand of $V_{N}$. Let $V^{*}$ be a $G$-composition factor of $V$ such that $\left(V^{*}\right)_{N}$ has a summand $U^{*}$ isomorphic with $U_{N}$. Using assumption (ii) and the fact that $N$ is abelian, we have $[M, N]=[K, N] \leqslant K \cap N=\operatorname{ker}\left(N\right.$ on $\left.U^{*}\right)$. In other words, $M$ centralizes $N / \operatorname{ker}\left(N\right.$ on $\left.U^{*}\right)$ and is therefore contained in the stabilizer of the homogeneous component of $\left(V^{*}\right)_{N}$ containing $U^{*}$. Suppose that this stabilizer is the group G. Then $K \cap N=\operatorname{ker}\left(N\right.$ on $\left.U^{*}\right) \triangleleft G$, and so by assumption (iii) we have $K \cap N$ $=1$. In that case $[K, N]=1$, and we have $M \leqslant C_{G}(N)$. Because $N \& Z(G)$, by the maximality of $M$ we have $M=C_{G}(N) \triangleleft G$, contradicting the hypothesis that $M$ is not normal in $G$. Therefore the stabilizer of the homogeneous component of $\left(V^{*}\right)_{N}$ containing $U^{*}$ is precisely $M$, and by Clifford's theorem applied to $V^{*}$ we have $\operatorname{dim}_{k} V^{*} \geqslant|G: M| \operatorname{dim}_{k} U^{*}=|G: M| \operatorname{dim}_{k} U=\operatorname{dim}_{k} V$ by definition of an induced module. Hence $V^{*}=V$ and $V$ is irreducible, as claimed.

We conclude the section by noting some special circumstances under which the hypotheses of (1.3) are satisfied; verification is straightforward and is left to the reader.

(1.4) Let $G$ be a finite, soluble group with a unique minimal normal subgroup $N$. Assume that $N$ is complemented in $G$, by $H$ say. Let $L$ be a nonnormal, maximal subgroup of $H$ such that $[N, L]<N$, and let $N_{1}$ be a maximal subgroup of $N$ containing $[N, L]$. Set $M=L N$ and $K=L N_{1}$. Then $M$ is a nonnormal, maximal subgroup of $G$ containing $N, K$ is a normal subgroup of $M$, and

(i) $M / K$ is cyclic,

(ii) $N K=M$, and

(iii) $\operatorname{core}_{G}(K)=1$. 
2. Multiprimitive groups. A group is said to be primitive, in the sense of the theory of permutation groups, if it has a faithful, primitive permutation representation. It is well known that a finite soluble group $G$ is primitive if and only if it has a self-centralizing, minimal normal subgroup, or, equivalently, if and only if its Fitting subgroup is a minimal normal subgroup. If we write $\operatorname{soc}(G)$ for the socle of a group, i.e. the product of all its minimal normal subgroups, $\operatorname{soc}(G)$ is itself a minimal normal subgroup when $G$ is a primitive, soluble group and is complemented in $G$ by a maximal subgroup $H$ (say). Furthermore $\operatorname{soc}(G)$ is then an elementary abelian $p$-group for some prime $p$ and may be regarded as an irreducible $\mathrm{Z}_{p} H$-module, faithful for $H$. Primitive groups are important in the theory of finite, soluble groups for several reasons. One is that a minimal counterexample in a 'reductio ad absurdum' proof often turns out to be primitve; the theory of Schunck classes owes a lot to this observation. Another is that primitive groups are relatively easy to construct and analyse: if $H$ is a group which possesses a faithful, irreducible module $V$ over some prime field $\mathbf{Z}_{p}$ (for example, $H$ any primitive group with $p \gamma|\operatorname{soc}(H)|$ ), the semidirect product $[V] \cdot H$, formed in the usual way by writing $V$ multiplicatively and regarding $H$ as a group of operators, is then a primitive group.

It is easy to see that an arbitrary finite group $H$ appears both as a subgroup and an epimorphic image of a primitive group. For choose primes $p, q$ such that $p$ divides $q-1$, let $X$ be the nonabelian group of order $p q$, and let $W=X \sim_{\text {reg }} H$, the standard wreath product with respect to the regular permutation representation of $H$. Then it follows easily from the Lemma of [4] that $W$ is a primitive group. But the main object of this section is to prove a stronger embedding theorem, namely that an arbitrary finite, soluble group $H$ can be embedded in a soluble group $G$ which has every epimorphic image primitive. If $X$ denotes a class of groups and $Q$ the usual closure operation of taking quotients, the class $X^{Q}$ is by definition the largest $Q$-closed class within $X$. Let $G$ denote the class of all finite, soluble groups, $\mathbb{P}$ the class of primitive groups in $G$ together with all groups of order 1 (since by convention all classes contain groups of order 1), and $s$ the closure operation of taking subgroups. Then the theorem we are aiming to prove may be restated thus: $s\left(\Re^{Q}\right)=\mathfrak{G}$.

There are many ways of characterising the groups in $\mathfrak{P Q}$, and as a sample we offer:

(2.1) Each of the following is necessary and sufficient for a nontrivial group $G$ to belong to $\Re Q$ :

(a) $G$ has a unique chief series and all its chief factors are complemented;

(b) The lower nilpotent series of $G$ is a chief series;

(c) The upper nilpotent series of $G$ is a chief series;

(d) $G$ is extreme (cf. Definition 3 of [1]) and $G$ has prefrattini subgroups of order 1 . 
The proofs of this and the next two remarks are elementary and are omitted.

(2.2) Let $N$ be a minimal normal subgroup of a group $G$ and assume that $G / N \in \mathfrak{Q Q}$. Let $S / N=\operatorname{soc}(G / N)$, and assume that $[N, S] \neq 1$. Then $G \in \mathfrak{B Q}$.

(2.3) Let $K$ and $L$ be normal subgroups of a group in $\mathrm{BQ}$ and assume that $K \leqslant L$. Then $K$ is complemented in $L$, i.e. $L$ has a subgroup $H$ such that $K H=L$ and $K \cap H=1$.

I shall give way to the temptation of suggesting a name for groups in $\$ Q$ by calling them multiprimitive. The notation $C(n)$ will denote a cyclic group of order $n$, and $E(n / q)$ will denote a primitive group $E$ satisfying (i) $E / \operatorname{soc}(E) \cong$ $C(n)$ and (ii) the prime $q$ divides $|\operatorname{soc}(E)|$. It can be shown that a group $E(n / q)$ exists, provided that $q$ does not divide $n$, and that then $|\operatorname{soc}(E(n / q))|=q^{m}$, where $m$ is the order of $q(\bmod n)$ (cf. Satz 3.10 in Chapter II of [6]). If $G$ is a nonabelian, multiprimitive group, it is easy to see that $G / G^{\prime \prime}$ is isomorphic with an $E(p / q)$ for suitable primes $p, q$. If $\pi$ is a set of primes, denote by $\mathcal{G}_{\pi}$ the class of finite, soluble $\pi$-groups. If $G$ is a finite group, let $\sigma(G)$ denote the set of primes which divide its order. The main result of this section is the following theorem:

THEOREM 1. Let $G$ be a finite, soluble group, let $\sigma=\sigma(G)$, and assume that $|\sigma| \geqslant 2$. Then there exists a group $H$ in $\Re \mathrm{Q} \cap \mathrm{G}_{\sigma}$ such that $H$ contains a subgroup isomorphic with $G$.

Let $\sigma=\sigma(G)=\left\{p_{0}, p_{1}, \ldots, p_{t-1}\right\}$. For each natural number $m$, let $[m]$ denote the remainder on dividing $m$ by $t \quad(=|\sigma|)$; thus $0 \leqslant[m] \leqslant t-1$. Define a group $W_{m}$ recursively as follows: $W_{0}=C\left(p_{0}\right)$ and $W_{m}=W_{m-1}$ $\sim_{\text {reg }} C\left(p_{[m]}\right)$ for $m \geqslant 1$. By I, 15.9 of [6] the group $G$ is isomorphic with a subgroup of $W_{m}$ for sufficiently large $m$. Therefore Theorem 1 is a consequence of the following:

(2.4) For an arbitrary natural number $m$ the group $W_{m}$ is isomorphic with a subgroup of a group belonging to $\Re \mathrm{Q} \cap \mathrm{G}_{\sigma}$.

The key to proving (2.4) is the following result, which shows how, in favourable circumstances, two multiprimitive groups can be fitted together to make a third.

(2.5) Let $X, G \in \mathbb{P Q}$. Let $N=\operatorname{soc}(G)$, let $Y=X^{\prime}$, and assume that the prime $|X: Y|=q$ (say) is the same as the prime dividing $|N|$. By (2.3) $X$ has a subgroup $Q$ of order $q$ such that $X=Y Q$, and $Q$ can clearly be regarded as $a$ subgroup of $\operatorname{Aut}(Y)$. Let $H$ be a complement to $N$ in $G$, assume that $H$ has a nonnormal, maximal subgroup $L$ such that $[N, L]<N$, and let $N_{1}$ be a maximal subgroup of $N$ containing $[N, L]$. Write $M=N L$ and $K=N_{1} L \quad(\triangleleft M)$. Since $M / K \cong C(q)$, there exists a homomorphism $\alpha: M \rightarrow \operatorname{Aut}(Y)$ such that 
$\operatorname{im}(\alpha)=Q$ and $\operatorname{ker}(\alpha)=K$. Then the twisted wreath product $W$ of $Y$ with $G$ for $(M, \alpha)$ is multiprimitive.

Proof. Let $T$ be a transversal to $M$ in $G$, and let $B$ as usual denote the base group of $W$. If $R$ is a subgroup of $Y$ and $S$ a subset of $T$, recall that $R(S)$ denotes the subgroup $\{(f, 1): f(t) \in R$ for $t \in S, f(t)=1$ for $t \in T \backslash S\}$ of $B$, and as a further simplification denote $R(T)$ by $R^{*}$; thus $B$ is $Y^{*}$ in this notation. Let

$$
1=R_{n}<R_{n-1}<\ldots<R_{1}<Y<X
$$

denote the unique chief series of $X$. The chief factor $U=Y / R_{1}$ of $X$ may be regarded as a nontrivial, irreducible $\mathrm{Z}_{p} Q$-module for some prime $p$ different from $q$ and therefore as an irreducible $\mathrm{Z}_{p} M$-module with $\operatorname{ker}(M$ on $U)=K$ (via $\alpha$ ). By (1.1) the normal factor $Y^{*} /\left(R_{1}\right)^{*}$ of $W$, considered by restriction as a $\mathrm{Z}_{p} G$-module, is isomorphic with $U^{G}$. By (1.3) and (1.4) $U^{G}$ is irreducible, and therefore $Y^{*} /\left(R_{1}\right)^{*}$ is a chief factor of $W$. Because $W / Y^{*} \cong G \in \Re Q$ and $N$ acts nontrivially on $Y^{*} /\left(R_{1}\right)^{*}$, by $(2.2)$ we have $W /\left(R_{1}\right)^{*} \in \Re^{Q}$.

Next we prove that $\left(R_{1}\right)^{*} /\left(R_{2}\right)^{*}$ is a chief factor of $W$ (provided that $n \geqslant$ 2). This done, it will then follow from (2.2) that $W /\left(R_{2}\right)^{*} \in \Re Q$. In fact, our proof will show equally well that $\left(R_{i}\right)^{*} /\left(R_{i+1}\right)^{*}$ is a chief factor of $W$ for $i \geqslant 1$, and so, applying (2.2) repeatedly down the series, we shall obtain $W \in \Re \mathrm{Q}$, as required. No generality is lost if we simplify notation by writing $R_{2}=1$ and $R$ for $R_{1}$, and then show that $R^{*}$ is a minimal normal subgroup of $W$. Let $A$ be a nontrivial, normal subgroup of $W$ contained in $R^{*}$. Ennumerate the transversal $T$ as $\left\{t_{1}=1, t_{2}, \ldots, t_{n}\right\}$, where $n=|G: M|$. When $f\left(t_{i}\right)=y_{i}$ for $i=1$, $\ldots, n$, write $\left(y_{1}, \ldots, y_{n}\right)$ for the element $(f, 1)$ of $B$. Let $1 \neq a=\left(r_{1}, \ldots\right.$, $\left.r_{n}\right) \in A, r_{i} \in R$, and for notational convenience suppose that $r_{1} \neq 1$. If $Z(Y)$ $\neq 1$, we have $R \leqslant Z(Y)$ since $Z(Y) \triangleleft X$ and $R$ is the unique minimal normal subgroup of $X$; but this is impossible because $R=C_{Y}(R)<Y$. Hence $Z(Y)=1$. Therefore there is a $y \in Y$ such that $y^{-1} r_{1} y \neq r_{1}$. Let $u=(y, 1, \ldots, 1)$. Then $A$ contains the element $a^{-1} u^{-1} a u=\left(a_{1}, 1, \ldots, 1\right)$, where $a_{1}=$ $r_{1}^{-1} y^{-1} r_{1} y$, a nontrivial element of $R$. Since $N \triangleleft G$, the $i$ th component $Y\left(t_{i}\right)$ of the base group is a normal subgroup of $B N$. Therefore $R\left(t_{1}\right)$ is also a normal subgroup of $B N$ because $\operatorname{soc}\left(Y\left(t_{1}\right)\right)$ is characteristic in $Y\left(t_{1}\right)$. Furthermore, $B N$ induces on $R\left(t_{1}\right)$ a group $\Gamma$ of automorphisms isomorphic with $\left[Y\left(t_{1}\right) / R\left(t_{1}\right)\right] \cdot$ $\left(N / N_{1}\right)$, which is in turn isomorphic with $X / R$. In fact, the semidirect product $\left[R\left(t_{1}\right)\right] \cdot \Gamma$ is easily seen to be isomorphic with $X$, and therefore $R\left(t_{1}\right)$ is a minimal normal subgroup of $B N$. Since $A \cap R\left(t_{1}\right)$ is a nontrivial, normal subgroup of $B N$, it follows that $R\left(t_{1}\right) \leqslant A$. But $G$ permutes the cosets of $M$ transitively, and hence $R\left(t_{i}\right) \leqslant A$ for $i=1, \ldots, n$. This shows that $R^{*} \leqslant A$ and therefore that $R^{*}$ is a minimal normal subgroup of $W$. The conclusion of the proof is now as indicated above. 
Proof of (2.4). First suppose that $|\sigma|=2$. Write $p_{0}=p$ and $p_{1}=q$, so that $W_{0}=C(p), W_{1}=C(p) \sim_{\mathrm{reg}} C(q), W_{2}=\left(C(p) \sim_{\mathrm{reg}} C(q)\right) \sim_{\mathrm{reg}} C(p)$, etc. Assume for the moment that $i \geqslant 0)$ is even and that $X_{i}$ is a group in $\sharp Q \cap \subseteq_{\{p, q\}}$ such that $X_{i} /\left(X_{i}\right)^{\prime} \cong C(q)$. We shall then construct a group $X_{i+1}$ in ßQ $\cap \mathcal{G}_{\{p, q\}}$ such that (i) $X_{i+1} /\left(X_{i+1}\right)^{\prime} \cong C(p)$ and (ii) $\left(X_{i+1}\right)^{\prime}$ contains a subgroup isomorphic with $\left(X_{i}\right)^{\prime} \sim_{\text {reg }} C(q)$. The construction of $X_{i+1}$ from $X_{i}$ when $i$ is odd will be the same with the roles of $p$ and $q$ interchanged. The derived group of a group $E(q / p)$ contains a copy of $W_{0}$. If we take $X_{0}=$ $E(q / p)$, the group $\left(X_{1}\right)^{\prime}$ will contain a subgroup isomorphic with $\left(X_{0}\right)^{\prime} \sim_{\text {reg }} C(q)$ and therefore certainly a subgroup isomorphic with $W_{1}$. By induction it will clearly follow that $\left(X_{m}\right)^{\prime}$ contains a subgroup isomorphic with $W_{m}$, and we shall have a proof of (2.4) in this case. Thus, given $X_{i}$, it remains to construct a group $X_{i+1}$ with the stated properties.

Let $E=E(p / q)$. Let $U$ be an irreducible $\mathrm{Z}_{p} E$-module, faithful for $E$, and let $D$ be the semidirect product $[U] \cdot E$. By (3.4) of [5] there exists an irreducible $\mathrm{Z}_{q} D$-module $V$, faithful for $D$, such that $[V, E]<V$. Let $C$ denote the semidirect product $[V] \cdot D$. It is clear that $C$ is a multiprimitive group of derived length 4 with $C / C^{\prime} \cong C(p)$. Set $M=V E$. It is straightforward to confirm that, with $\left(X_{i}, C, V, D, E, M\right)$ in place of $(X, G, N, H, L, M)$, the hypotheses of (2.5) are satisfied. Accordingly, if we define $X_{i+1}$ to be the group $\left(X_{i}\right)^{\prime} \sim_{(M, \alpha)} C$ constructed there, we have $X_{i+1} \in \mathcal{B Q} \cap \mathcal{G}_{\{p, q\}}$ and $X_{i+1} /\left(X_{i+1}\right)^{\prime} \cong C(p)$. We must show that $\left(X_{i+1}\right)^{\prime}$ contains a subgroup isomorphic with $\left(X_{i}\right)^{\prime} \sim_{\text {reg }} C(q)$. Recall the description of the twisted wreath product given in $\S 1$. Since $U M=$ $G$ and $U \cap M=1$, we may take $T=U$ for the transversal to $M$ in $G$. Let $g \in$ $\operatorname{soc}(E), g \neq 1$. Since $g$ has order $q$ and normalizes but does not centralize the subgroup $U$, there exists a subset $U_{0}=\left\{u_{1}, \ldots, u_{q}\right\}$ of $U$ such that $g^{-1} u_{i} g=$ $u_{i+1}, i=1, \ldots, q-1$, and $g^{-1} u_{q} g=u_{1}$. We assert that, writing $Y$ for $\left(X_{i}\right)^{\prime}$, we have

$$
\left\langle Y\left(U_{0}\right), g\right\rangle \cong\left(X_{i}\right)^{\prime} \sim_{\text {reg }} C(q) .
$$

Since $u_{i} g=g u_{i+1}$ for $i=1, \ldots, q$ (reducing suffices modulo $q$ ), direct calculation from equation $(1, \beta)$ shows that

$$
(e, g)^{-1}(f, 1)(e, g)=\left(f^{*}, 1\right),
$$

where $f^{*}\left(u_{i}\right)=f\left(u_{i+1}\right)^{g^{-1}}=f\left(u_{i+1}\right)$ since $g \in E \leqslant \operatorname{ker}(\alpha)$. But equation $(2, \beta)$ implies that $g$ permutes the direct components $Y\left(u_{1}\right), \ldots, Y\left(u_{q}\right)$ of $Y\left(U_{0}\right)$ in a cycle of length $q$, whence the isomorphism of $(2 . \alpha)$ is clear.

When $|\sigma| \geqslant 3$, the recursive construction of a group $X_{m} \in \Re Q \cap \mathcal{G}_{\sigma}$ such that $\left(X_{m}\right)^{\prime}$ contains a copy of $W_{m}$ is even easier. Let $([m-1],[m])=(i, j)$; accordingly $W_{m}=W_{m-1} \sim_{\mathrm{reg}} C\left(p_{j}\right)$. Assume inductively that a group $X_{m-1}$ 
has already been constructed satisfying the following conditions:

(a) $X_{m-1} \in \Re Q \cap G_{\sigma}$;

(b) the prime $p_{i}$ divides $\left|\left(X_{m-1}\right)^{\prime}:\left(X_{m-1}\right)\right|$;

(c) the prime $\left|X_{m-1}:\left(X_{m-1}\right)^{\prime}\right| \notin\left\{p_{i}, p_{j}\right\}$;

(d) $\left(X_{m-1}\right)^{\prime}$ contains a subgroup isomorphic with $W_{m-1}$.

To start the induction, choose $q \in \sigma \backslash\left\{p_{0}, p_{1}\right\}$ and take $X_{0}=E\left(q / p_{0}\right)$. The construction of $X_{m}$ from $X_{m-1}$ proceeds as follows. Let $k=[m+1]$ and choose $r \in \sigma \backslash\left\{p_{j}, p_{k}\right\}$. Let $E=E\left(r / p_{j}\right)$, and let $D$ be a primitive group such that $D / \operatorname{soc}(D) \cong E$ and $\operatorname{soc}(D)$ is a $q$-group, where $q=\left|X_{m-1}:\left(X_{m-1}\right)^{\prime}\right|$. If $R$ is a Sylow $r$-subgroup of $E$, we can arrange that $[\operatorname{soc}(D), R]<\operatorname{soc}(D)$ by (3.4) of [5] (in fact, it is easy to see from Clifford's theorem that this holds in any case), and with $M=\operatorname{soc}(D) R$ we can construct the multiprimitive group $X_{m}=$ $\left(X_{m-1}\right)^{\prime} \sim_{(M, \alpha)} D$ according to the recipe of (2.5). It is obvious that $X_{m}$ fulfils requirements (a), (b), and (c) above. If $P$ is the Sylow $p_{j}$-subgroup of $E$, we can choose $P$ as the transversal to $M$ in $D$. If $B$ denotes as usual the base group of $X_{m}$, by (1.2) $B P \cong\left(X_{m-1}\right)^{\prime} \sim_{\text {reg }} P$, which by elementary properties of a wreath product contains a copy of $\left(X_{m-1}\right)^{\prime} \sim_{\text {reg }} C\left(p_{j}\right)$. Since $B P \leqslant\left(X_{m}\right)^{\prime}$, requirement (d) is also satisfied, and it is now clear by induction that $\left(X_{m}\right)^{\prime}$ contains a subgroup isomorphic with $W_{m}$. This completes the proof of (2.4) and with it the proof of Theorem 1.

3. A conjecture of Dade's. Let $\gamma(X)$ denote the composition length of a group or a module $X$, i.e. the number of factors in a composition series of $X$ (provided, of course, that it has one); the Jordan-Hölder theorem guarantees that $\gamma(X)$ is an invariant of $X$. Let $G$ be a finite, soluble group and $C$ a Carter subgroup of $G$. Thompson conjectured in [9], and Dade subsequently proved in [3], that the nilpotent length $l(G)$ of $G$ is bounded as a function of $c=\gamma(C)$; explicitly, he shows that

$$
l(G) \leqslant 10\left(2^{c}-1\right)-4 c .
$$

Although the methods used in obtaining this result are of great interest, they do not pretend to give a realistic estimate for $l(G)$, as Dade himself points out. In fact, he goes on to suggest that it ought to be possible to find a constant $K$ such that $l(G) \leqslant K \gamma(C)$. I should like to propose, perhaps rather rashly, that this inequality may even hold with $K=2$. By slightly modifying the methods used by Seitz in [7], I can show that this is the case when $G$ is an $A$-group (i.e. a finite, soluble group all of whose Sylow subgroups are abelian), an observation that has also been made by Seitz in an as yet unpublished work [8]; and it has also been confirmed in several other limited special cases. But no attempt is made to consider this question here. Rather the aim of this section is simply to show that $K=2$ cannot be bettered, even for $A$-groups. 
First we prove a result which provides some information about the Carter subgroup of a certain wreath product.

(3.1) Let $H$ be a nilpotent $\pi$-group, $K$ a soluble $\pi^{\prime}$-group, $H$ and $K$ both finite. Let $C$ be a Carter subgroup of $K$. If $W=K \sim_{\rho} H$, the wreath product of $K$ with $H$ for a transitive permutation representation $\rho$ of $H$, then $H \times \Delta(C)$ is a Carter subgroup of $W$, where $\Delta(C)$ denotes the diagonal of $C$ in the base group.

Proof. Let $B$ denote the base group of $W$. Since $W / B \cong H$ and is nilpotent, a Carter subgroup of $W$ covers $W / B$ and therefore contains a Hall $\pi$-subgroup of $W$, i.e. a conjugate of $H$. Therefore $H$ is a Hall $\pi$-subgroup of some Carter subgroup $D$ (say) of $W$; it follows that $D$ is a Carter subgroup of $H C_{W}(H)$ $=H \times C_{B}(H)$ and that all Carter subgroups of $H C_{W}(H)$ are Carter subgroups of $W$. Because $\rho$ is transitive, we have $C_{B}(H)=\Delta(K)=\{(k, k, \ldots, k): k \in K\}$, the diagonal subgroup of $B$. Clearly $\Delta(C)$ is a Carter subgroup of $\Delta(K)$, and, since a Carter subgroup of a direct product is the product of Carter subgroups of the direct factors, it follows that $H \times \Delta(C)$ is a Carter subgroup of $H C_{W}(H)$ and hence of $W$.

Theorem 2. Let $n$ be a positive integer. There exists an A-group $G$ which has a Carter subgroup $C$ such that $l(G)=2 \gamma(C)=2 n$.

Proof. Choose a sequence of $2 n+1$ distinct primes $p_{1}, q_{1}, \ldots, p_{n}$, $q_{n}, p_{n+1}$ such that $p_{i}$ divides $q_{i}-1$ and $q_{i}$ divides $p_{i+1}-1$ for $i=1, \ldots, n$; that such a choice is possible follows from Dirichlet's theorem on primes in arithmetic progression. Let $E_{i}=E\left(p_{i} / q_{i}\right)$, the nonabelian group of order $p_{i} q_{i}$, and let $P_{i}, Q_{i}$ denote respectively a Sylow $p_{i}$-subgroup and the normal Sylow $q_{i}$-subgroup of $E_{i}$. Let $U_{i}$ be a 1-dimensional, nontrivial $\mathrm{Z}_{p_{i+1}} Q_{i}$-module and let $V_{i}=\left(U_{i}\right)^{E_{i}}$. Denoting the semidirect product $\left[V_{i}\right] \cdot E_{i}$ by $G_{i}$, we note that $G_{i}$ is a multiprimitive $A$-group of order $p_{i+1}^{p_{i}} q_{i} p_{i}$, that $\left(V_{i}\right)_{P_{i}}$ is isomorphic with the regular module $Z_{p_{i+1}} P_{i}$ and therefore that the subgroup $\left[V_{i}, P_{i}\right]$ has index $p_{i+1}$ in $V_{i}$. Let $M_{i}$ denote the nonnormal, maximal subgroup $V_{i} P_{i}$ of $G_{i}$ and observe that $M_{i}$ has $\left[V_{i}, P_{i}\right] P_{i}$, $=K_{i}$ say, as a maximal, normal subgroup of index $p_{i+1}$.

Let $1 \leqslant i \leqslant n$ and let $\pi_{i}=\left\{p_{i}, q_{i}, p_{i+1}, q_{i+1}, \ldots, p_{n}, q_{n}, p_{n+1}\right\}$. Suppose that a group $X_{i}$ exists with the following properties:

(a) $X_{i}$ is an $A$-group belonging to $\$ \mathrm{Q} Q \cap \mathrm{\Xi}_{\pi_{i}}$;

(b) if $Y_{i}$ denotes $\left(X_{i}\right)^{\prime}$, then $X_{i} / Y_{i} \cong C\left(p_{i}\right)$ and $Y_{i}$ is a normal $p_{i}$-complement of $X_{i}$;

(c) $Y_{i}$ has a Carter subgroup $C_{i}$ of order $q_{i} q_{i+1} \ldots q_{n}$;

(d) $l\left(Y_{i}\right)=2(n-i+1)$.

It is straightforward to verify that, for $i=n$, these conditions hold with $X_{n}=$ $G_{n}$. So, given $X_{i}$, we shall construct a group $X_{i-1}$ which also satisfies conditions 
(a) - (d). Then by induction on the suffix $i$ (with the integers in their reverse order) we shall obtain a group $X_{1}$ fulfilling these conditions and, in particular, a group $Y_{1}$ of nilpotent length $2 n$ whose Carter subgroup has order $q_{1} q_{2} \ldots$ $q_{n}$ and hence composition length $n$. The construction of $X_{i-1}$ is given by (2.5) with $\left(X_{i}, G_{i-1}, V_{i-1}, E_{i-1}, P_{i-1}, M_{i-1}, K_{i-1}\right)$ in place of $(X, G, N, H, L, M, K)$, in other words $X_{i-1}$ is the twisted wreath product of $Y_{i}$ with $G_{i-1}$ for $\left(M_{i-1}, \alpha_{i-1}\right)$, where $\alpha_{i-1}$ is a suitable homomorphism from $M_{i-1}$ onto $P_{i}$ with kernel $K_{i-1}$ (identifying the Sylow $p_{i}$-subgroup $P_{i}$ of $X_{i}$ with a subgroup of $\left.\operatorname{Aut}\left(Y_{i}\right)\right)$.

We now proceed to verify that $X_{i-1}$ satisfies conditions (a)-(d) with $i-1$ in place of $i$. Since the class of $A$-groups is closed under direct products, the group $X_{i-1}$ is the semidirect product of an $A$-group $B_{i-1}$, by definition the base group of the wreath product, with an $A$-group $G_{i-1}$ of coprime order; thus $X_{i-1}$ is an $A$-group. Since $X_{i-1}$ is obviously a $\pi_{i-1}$-group and by (2.5) belongs to $\Re \mathrm{Q}$, condition (a) certainly holds. Since condition (b) is obviously satisfied, we move on to condition (c). Noting that $V_{i-1}$ is the nilpotent residual of $V_{i-1} Q_{i-1}$, we see that $B_{i-1} Q_{i-1}$, as an $\Re$-crucial, maximal subgroup of $Y_{i-1}$, contains a Carter subgroup of $Y_{i-1}$ (cf. Theorem (5.4) and the preceding Definition in [2] - $\Re$ denotes the class of nilpotent groups). Let $C_{i}$ be a Carter subgroup of $Y_{i}$. By (1.2) we have $B_{i-1} Q_{i-1}=Y_{i} \sim_{\text {reg }} Q_{i-1}$, and therefore by (3.1) a Carter subgroup of $B_{i-1} Q_{i-1}$ (and hence of $Y_{i-1}$ ) is isomorphic with $C_{i} \times Q_{i-1}$, whence condition (c) holds. Finally let

$$
1<F_{1}<\ldots<F_{l-1}\left(=Y_{i-1}\right)<F_{l}=X_{i-1}
$$

denote the unique chief series of $X_{i-1}$. Clearly $l=l\left(Y_{i}\right)+3$. By (2.1) this is the upper nilpotent series of $X_{i-1}$, and therefore $1<F_{1}<\ldots<F_{l-1}$ is the upper nilpotent series of $Y_{i-1}$. Hence $l\left(Y_{i-1}\right)=l-1=l\left(Y_{i}\right)+2=$ $2(n-[i-1]+1)$, and the verification of the induction step is complete.

\section{REFERENCES}

1. R. W. Carter, B. Fischer and T. O. Hawkes, Extreme classes of finite soluble groups, J. Algebra 9 (1968), 285-313. MR 37 \#161.

2. R. W. Carter and T. O. Hawkes, The $\mathfrak{F}$-normalizers of a finite soluble group, J. Algebra 5 (1967), 175-202. MR 34 \#5914.

3. E. C. Dade, Carter subgroups and Fitting heights of finite solvable groups, Illinois J. Math. 13 (1969), 449-514. MR 41 \#339.

4. T. O. Hawkes, An example in the theory of soluble groups, Proc. Cambridge Philos. Soc. 67 (1970), 13-16. MR 40 \#1477.

5. - The family of Schunck classes as a lattice, J. Algebra (to appear) (available in preprint form from the University of Warwick).

6. B. Huppert, Endliche Gruppen. I, Die Grundlehren der math. Wissenschaften, Band 134, Springer-Verlag, Berlin and New York, 1967. MR 37 \#302.

7. G. M. Seitz, Solvable groups having system normalizers of prime order, Trans. Amer. Math. Soc. 183 (1973), 165-173. 
8. G. M. Seitz, On system normalizers of prime order, University of Oregon (preprint).

9. J. G. Thompson, Automorphisms of solvable groups, J. Algebra 1 (1964), 259267. MR 30 \#3920.

DEPARTMENT OF MATHEMATICS, MICHIGAN STATE UNIVERSITY, EAST LANSING, MICHIGAN 48824

Current address: Mathematics Institute, University of Warwick, Coventry CV4 7AL, England 